\title{
Predictors of Recurrent Hospital Admission for Patients Presenting With Diabetic Ketoacidosis and Hyperglycemic Hyperosmolar State
}

\author{
Annabel L. Bradford ${ }^{\mathrm{a}, \mathrm{d}}$, Courtney Champagne Crider ${ }^{\mathrm{b}}$, Xizheng $\mathrm{Xu}^{\mathrm{c}}$, \\ Syed Hasan Naqvic
}

\begin{abstract}
Background: Diabetic ketoacidosis (DKA) and hyperglycemic hyperosmolar state (HHS) are two serious, preventable complications of diabetes mellitus. Analysis of variables associated with recurrent DKA and HHS admission has the potential to improve patient outcomes by identifying possible areas for intervention. The aim of this study was to evaluate potential predictors of recurrent DKA or HHS admission.
\end{abstract}

Methods: This was a retrospective case-control study of 367 patients presenting during a 5-year period with DKA or HHS at a US tertiary academic medical center. Six potential readmission risk factors identified via literature review were coded as " 1 " if present and " 0 " if absent. Readmission odds ratios (ORs) for each risk factor and for the combined score of significant risk factors were calculated by logistic regression.

Results: Readmission odds were significantly increased for patients with age $<35$, history of depression or substance/alcohol abuse, and self-pay/publicly funded insurance. HbA1C $>10.6 \%$ on admission and ethnic minority status did not significantly increase readmission odds, with inadequate study power for these variables. A total "ABCD" score, based on Age ( $<35$ years), Behavioral health (depression), insurance Coverage (self-pay/publicly funded insurance), and Drug/alcohol abuse, also had a significant effect on readmission odds.

Conclusions: Consideration of individual risk factors and the use of a scoring system based on objective predictors of recurrent DKA and HHS admission could be of value in helping identify patients with high readmission risk, allowing interventions to be targeted most effectively to reduce readmission rates, associated morbidity, and mor-

Manuscript accepted for publication October 21, 2016

aDepartment of Psychiatry, Maine Medical Center, 22 Bramhall St., Portland, ME 04102, USA

bDepartment of Dermatology, Saint Louis University, 1755 South Grand Boulevard, St. Louis, MO 63104, USA

'University of Missouri School of Medicine, One Hospital Drive, Columbia, MO 65212, USA

${ }^{\mathrm{d}}$ Corresponding Author: Annabel L. Bradford, Department of Psychiatry, Maine Medical Center, 22 Bramhall St., Portland, ME 04102, USA.

Email: abradford@mmc.org

doi: https://doi.org/10.14740/jocmr2792w tality.

Keywords: Diabetes mellitus; Health care quality; Hospital admission

\section{Introduction}

Diabetic ketoacidosis (DKA) and hyperglycemic hyperosmolar syndrome (HHS) are two of the most serious acute complications of diabetes mellitus. The National Diabetes Surveillance Program of the Centers for Disease Control (CDC) estimated that there were 140,000 hospital discharges for DKA in 2009 in the United States, compared to 62,000 in 1980 [1]. Given the $226 \%$ increase in DKA hospitalizations, it is crucial for hospitals and physicians to attend to DKA prevention. During this same period, deaths from hyperglycemic crisis (including DKA and HHS) decreased $19.8 \%$ (from 3,012 in 1980 to 2,417 in 2009) [2]. Despite the overall decrease in deaths from hyperglycemic crisis, the high relative mortality associated with HHS (5-20\% for HHS vs. $<5 \%$ for DKA) [3] underlines the importance of HHS prevention in improving patient outcomes.

A literature review of risk factors for DKA and HHS suggests potential objective predictors of disease recurrence that could assist physicians and hospitals in managing DKA and HHS patients to help prevent readmission. For example, younger age at DKA onset has been associated with increased risk of DKA recurrence $[3,4]$. Other studies note that readmitted DKA patients are more often male (58\%) than female $(52 \%)$ [5], while a rising trend in DKA readmission among female diabetic patients less than 35 years of age has also been noted [6].

In addition to the possible associations of age and gender with diabetic crisis readmission, studies suggest that baseline glucose management and patient comorbidities may play a role. Poor baseline glycemic control and elevated hyperglycated hemoglobin (HbA1C) have been associated with recurrent DKA [3, 7], with admitted DKA patients tending to have an $\mathrm{HbA} 1 \mathrm{C}$ greater than $10.6 \%$ [8] and $\mathrm{HbAlC}$ greater than $10.8 \%$ associated with a particularly high rate of readmission [9]. Among patient comorbidities, depression and alcohol or substance abuse seem to play strong roles [3]. In one study, patients with recurrent DKA admissions were more likely to have a history of depression than were their non-readmitted 
counterparts (53.7\% vs. 37\%) [4]. Alcohol and drug abuse were also associated with DKA readmission: $39.6 \%$ of readmitted patients abused alcohol (vs. 24.7\% of non-readmitted patients) and $51.7 \%$ used illicit drugs (vs. $22.5 \%$ of non-readmittees) [4]. In another study, $70 \%$ of drug users with DKA had more than one hospital admission, while only $15.9 \%$ of non-drug users with the same conditions were readmitted [10]. Alcohol and cocaine abuse have both been identified as risk factors for DKA and HHS admission [11], while active cocaine use may have a particularly strong association with DKA readmission $[12,13]$.

Socioeconomic factors also seem to be linked to readmission likelihood among patients with diabetes. Ethnic minority status and use of publicly funded health insurance have been linked to hospitalization and readmission for DKA [14]. Underinsurance also appears to be a risk [7]. Psychosocial, economic, and behavioral factors, including financial constraint, stretching a limited insulin supply, and homelessness, have been associated with readmission in DKA patients $[3,4]$. Hispanic patients with type 1 diabetes mellitus were identified as being at particularly high risk for DKA readmission [15], and hospital admission and mortality rates for DKA and HHS have been noted to be higher in black patients than in white patients with diabetes [11].

Frequent monitoring of hospital readmissions has been identified as a valuable means of recognizing and addressing preventable quality-of-care problems in medicine [16]. Analysis of recurrent admissions for DKA and HHS has the potential to help identify factors that could assist hospitals and physicians in obviating future readmissions from these preventable complications of diabetes mellitus. Our study sought to examine potential predictors of recurrent admission for patients presenting with DKA or HHS and to formulate a scoring system to identify patients at high risk for readmission. If these patients could be easily identified, their in-hospital management could be more effectively targeted to prevent readmission and associated morbidity and mortality.

\section{Materials and Methods}

This was a retrospective case-control study conducted via chart review at a tertiary academic medical center. The study was approved by the Institutional Review Board at the study site, and given the chart review design, specific informed consent for the study was not required. Inclusion criteria for involvement in the study were admission to the medical center between 2008 and 2013 with a primary diagnosis of DKA or HHS based on ICD- 9 codes. Patients younger than 5 years old and those who died during their initial admission were excluded. Literature review was used to identify six potential risk factors for DKA or HHS readmission. These risk factors were examined and scored as absent ( 0 ) or present (1) for each patient. Specifically, patients received 1 point for the presence of each of the following risk factors: age $<35$ years, history of depression, $\mathrm{HbA} 1 \mathrm{C}>10.6 \%$ on admission, substance/alcohol abuse history, ethnic minority status, and self-pay/publicly funded insurance.

Each individual risk factor, as well as the total score of
Table 1. Demographic and Clinical Characteristics of Studied Participants

\begin{tabular}{ll}
\hline Characteristic & \\
\hline $\begin{array}{l}\text { Age in years, mean (range) } \\
\text { Diabetic complication }\end{array}$ & $39.3(5-88)$ \\
$\quad$ DKA, N (\%) & $355(96.7)$ \\
$\quad$ HHS, N (\%) & $12(3.3)$ \\
Sex & $180(49.0)$ \\
$\quad$ Male, N (\%) & $187(51.0)$ \\
$\quad$ Female, N (\%) & $10.86(5.1-19.1)$ \\
\hline HbA1C on admission, mean (range)
\end{tabular}

significant individual risk factors, was compared for those patients readmitted for DKA or HHS during the 5-year study period vs. those who were not readmitted during the same period. Logistic regression was then used to find odds ratios (ORs) for readmission and $\mathrm{P}$ values for each individual risk factor, as well as for the combined score of significant risk factors. Risk of readmission for DKA or HHS over the 5-year study period was calculated by ABCD score, based on Age $(<35$ years $)$, Behavioral health (depression), insurance Coverage (self-pay/ publicly funded insurance), and Drug/alcohol abuse.

\section{Results}

Our study included 367 patients (355 diagnosed with DKA and 12 with HHS). Of the study participants, $49 \%$ were male, and $51 \%$ were female. The mean age of study participants was 39.3 years, with a range of $5-88$ years old (Table 1). Of the 367 study participants, $42.0 \%$ met risk factor criteria for age (age $<35$ years), $24.3 \%$ had a history of depression, and $28.9 \%$ had a history of substance or alcohol abuse. Patients who were self-pay or on publicly funded insurance made up $67.0 \%$ of the study group, and $18.3 \%$ were of ethnic minority status. The average $\mathrm{HbA} 1 \mathrm{C}$ on admission for patients in the study was 10.86 , with a range of $5.1-19.1$ and with $51.8 \%$ of study participants meeting risk criteria for $\mathrm{HbA1C}(\mathrm{HbAlC}>10.6$ on admission). Lastly, $27.0 \%$ of the study group was readmitted for DKA or HHS during the 5-year study period.

We found significantly increased odds of readmission for DKA and HHS with four out of six risk factors: age $<35$ years (OR: 3.021; 95\% CI: 1.878 - 4.866; P < 0.0001), history of depression (OR: 3.465; 95\% CI: 2.085 - 5.758; P < 0.0001), substance/alcohol abuse history (OR: 2.828; 95\% CI: 1.736 - 4.606; $\mathrm{P}<0.0001$ ), and self-pay/publicly funded insurance (OR: $1.773 ; 95 \%$ CI: $1.051-2.99$; $\mathrm{P}=0.032$ ). A history of depression showed the greatest odds of readmission. Two variables were found not to significantly increase the odds of readmission for DKA and HHS in this study: HbA1C $>10.6 \%$ on admission (OR: 1.458; 95\% CI: $0.914-2.323 ; \mathrm{P}=0.1123$ ) and ethnic minority status (OR: 0.742; 95\% CI: $0.396-1.389$; $\mathrm{P}=0.3508$ ). However, this study was found to be inadequately powered to detect a potential significant increase in odds for 
Table 2. Readmission Odds by Study Variable and by Combined ABCD Score

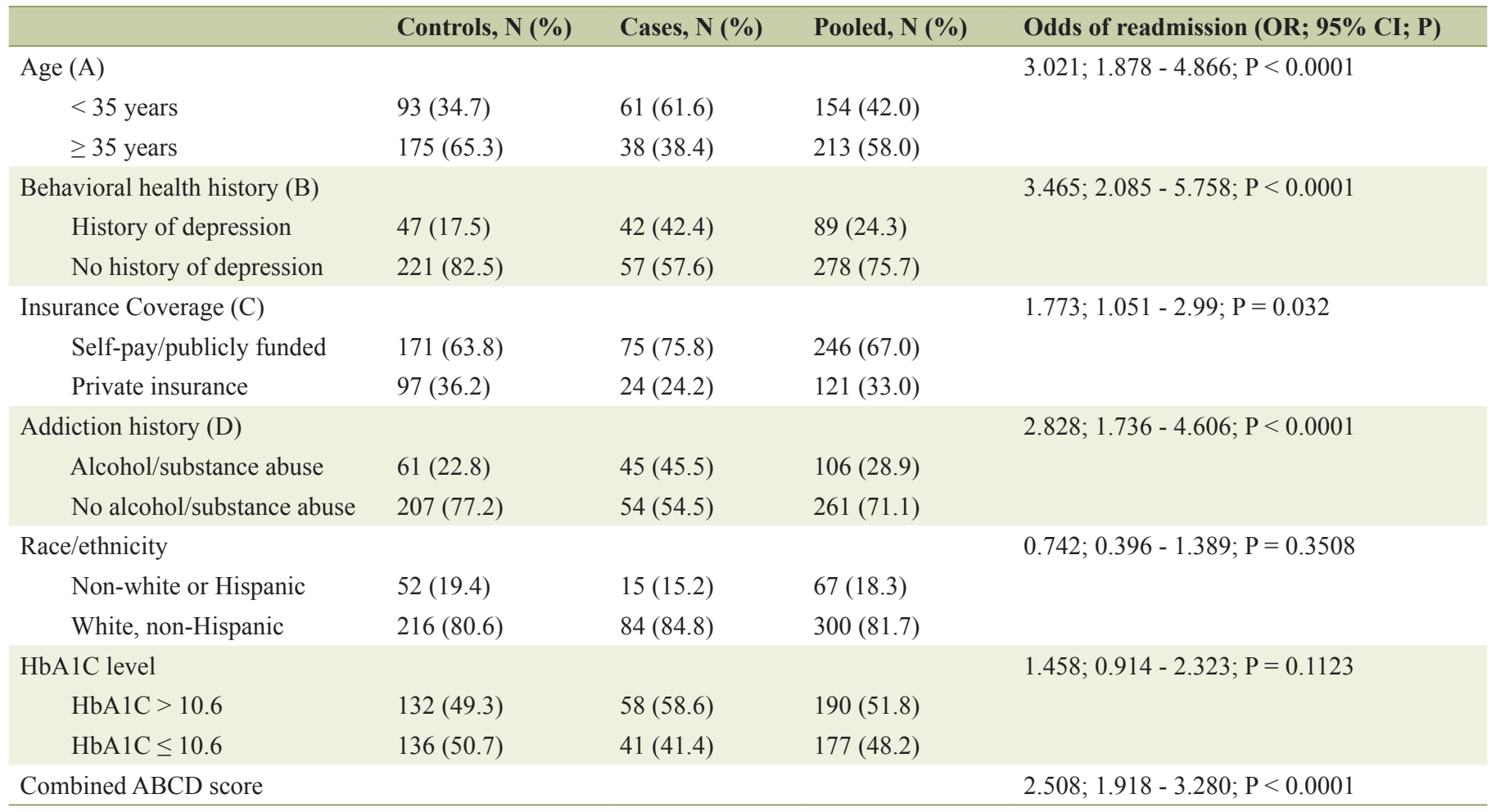

$\mathrm{HbA} 1 \mathrm{C}>10.6$ and ethnic minority status, for which the study was only powered at 0.437 and 0.148 , respectively (Table 2 ).

Using the variables found to exert a significant effect on readmission odds, a total "ABCD" score was calculated. This ABCD score was found to have a significant effect on odds of readmission (OR: 2.508; 95\% CI: 1.918 - 3.280; $\mathrm{P}<0.0001$ ). Risk of readmission for DKA or HHS also varied by ABCD score, with higher scores associated with higher readmission risks. Patients scoring 0 points on the ABCD scale had $11.1 \%$ readmission risk, while those scoring $1,2,3$, and 4 showed readmission risks of $14.9 \%, 27.1 \%, 52.7 \%$, and $86.7 \%$, respectively (Table 3 ).

\section{Discussion}

Among potential risk factors for readmission for DKA and HHS, younger age, comorbid behavioral health problems (es- pecially depression), poor baseline glucose control, drug and alcohol abuse, ethnic minority status, and self-pay or use of publicly funded health insurance are emphasized in the literature. The clear associations found in this study between readmission for DKA or HHS and young age $(<35$ years), behavioral health comorbidity (specifically, depression), self-pay/ publicly funded health insurance status, and drug and alcohol abuse support findings already described.

Younger age may be associated with an increased risk for readmission in our study and others via decreased patient experience with diabetes management, developmental issues, an end of parental oversight, and/or more irregular living arrangements or habits. Drug and alcohol abuse and behavioral health problems like depression, in turn, may exert their effects by interfering with treatment engagement, self-efficacy, and self-care. Self-pay or publicly funded health insurance status may increase readmission rates, in turn, by limiting access to healthcare. Patients with poor healthcare access are less likely

Table 3. Readmission Risk by ABCD Score

\begin{tabular}{lllll}
\hline ABCD score & Controls, $\mathbf{N}$ & Cases, $\mathbf{N}$ & Pooled, $\mathbf{~}$ & Readmission risk, $\%$ \\
\hline 0 & 40 & 5 & 45 & 11.1 \\
1 & 114 & 20 & 134 & 14.9 \\
2 & 86 & 32 & 118 & 27.1 \\
3 & 26 & 29 & 55 & 52.7 \\
4 & 2 & 13 & 15 & 86.7 \\
Any ABCD score $(0-4)$ & 268 & 99 & 367 & 27.0 \\
\hline
\end{tabular}


to be able to afford medication, to seek preventative treatment, and to engage in medically appropriate follow-up of health concerns.

Other risk factors for readmission identified in the literature were not found to exert significant effects in this study. Specifically, HbA1C on admission and ethnic minority status were not identified as significant risk factors for readmission. While these risk factors simply may not have mediated increased risk in our study, an absence of significant findings in this area may be due to inadequate power or other factors. Logically, higher baseline HbA1C levels should be associated with less adequate glucose management and increased readmission risk. In this study, the specific HbA1C cut-off used (greater than or less than 10.6) may have been too high to serve as a meaningful divide between patient groups with adequate versus inadequate pre-admission serum glucose control. If this study were redone, reanalysis of the effects of $\mathrm{HbA} 1 \mathrm{C}$ using a lower cut-off may yield different results.

This study also failed to find the significantly increased readmission risk identified in the literature for ethnic minority versus white patients. While ethnic minority status may not, in fact, consistently mediate increased readmission risk, a lack of significant findings in this area is likely due to insufficient study power (calculated as 0.148 ), since only $18.3 \%$ of study participants were identified as coming from ethnic minority groups. Repeating this study in a population with a greater representation of ethnic minority patients (and thus greater power for this analysis) may yield results more consistent with those found in other studies.

A consideration of recognizable risk factors for readmission and techniques for addressing these risk factors is very relevant to current efforts to improve patient care and to reduce healthcare costs. Many readmissions for DKA and HHS may be preventable. One study of scheduled and unscheduled hospital readmissions for diabetic patients used the Agency for Healthcare Research and Quality's Prevention Quality Indicators to estimate that one in five diabetic readmissions over a 3-month period was preventable [14]. Prevention of DKA and HHS through tighter glycemic control and facilitation of adherence to medical recommendations may be key in reducing hospital readmission rates for these diabetic complications.

Consideration of significant individual risk factors and/or use of a scoring system based on multiple objective predictors of recurrent DKA or HHS admission could be used to target interventions to patients at high risk for readmission. By combining the significant risk factors for DKA and HHS readmission identified in this study, an "ABCD" score (A: age; B: behavioral health; $\mathrm{C}$ : coverage; $\mathrm{D}$ : drug/alcohol abuse) could be calculated for patients admitted for DKA or HHS to help identify those with high readmission risk. Promising interventions could include intensified pre-discharge diabetic education, collaboration with psychiatric providers to ensure adequate treatment of behavioral health comorbidities, emphasis on affordable medications, closer post-discharge follow-up at easily accessible sites, and other more patient-friendly care regimens.

The main limitations of this study are small sample size (367 patients) and the limited time frame of chart review (5 years), as patient readmissions before or after this 5-year period could have been missed. Also, by using ICD-9 codes to identify study patients, individuals who were miscoded or misidentified in their admission or discharge notes may have been missed. Admission and discharge notes for those patients included in the study were reviewed to ensure that they were admitted for DKA or HHS.

DKA and HHS are two serious but largely preventable acute complications of diabetes mellitus. Analysis of recurrent admissions has the potential to help identify factors that could assist physicians and hospitals in obviating readmissions for these complications. In our study, age $<35$ years, history of depression or substance/alcohol abuse, and self-pay/publicly funded insurance status accurately predicted odds of hospital readmission for patients with DKA or HHS, both individually and as part of a combined "ABCD" score. The use of a scoring system based on these objective predictors of DKA and HHS readmission could be used to identify high-risk patients and to target interventions more effectively toward them, with the aim of preventing readmission and associated morbidity and mortality.

\section{Disclosures}

There is no grant support or other assistance to declare for this project, and the authors have no conflicts of interest to declare.

\section{References}

1. CDC. Diabetes Data \& Trends. DHHS Centers for Disease Control and Prevention. [Online] May 17, 2012. [Cited: September 2, 2013.] http://www.cdc.gov/diabetes/statistics/dkafirst/fig1.htm.

2. Wei NJ, Wexler DJ, Nathan DM, Grant RW. Intensification of diabetes medication and risk for 30-day readmission. Diabet Med. 2013;30(2):e56-62.

3. Liu CC, Chen KR, Chen HF, Huang HL, Ko MC, Li CY. Trends in hospitalization for diabetic ketoacidosis in diabetic patients in Taiwan: analysis of national claims data, 1997-2005. J Formos Med Assoc. 2010;109(10):725-734.

4. Kim H, Ross JS, Melkus GD, Zhao Z, Boockvar K. Scheduled and unscheduled hospital readmissions among patients with diabetes. Am J Manag Care. 2010;16(10):760767.

5. Steenkamp DW, Alexanian SM, McDonnell ME. Adult hyperglycemic crisis: a review and perspective. Curr Diab Rep. 2013;13(1):130-137.

6. Randall L, Begovic J, Hudson M, Smiley D, Peng L, Pitre N, Umpierrez D, et al. Recurrent diabetic ketoacidosis in inner-city minority patients: behavioral, socioeconomic, and psychosocial factors. Diabetes Care. 2011;34(9):1891-1896.

7. Nyenwe E, Loganathan R, Blum S, Ezuteh D, Erani D, Palace M, Ogugua C. Admissions for diabetic ketoacidosis in ethnic minority groups in a city hospital. Metabolism. 2007;56(2):172-178.

8. Umpierrez GE, Kelly JP, Navarrete JE, Casals MM, Kitabchi AE. Hyperglycemic crises in urban blacks. Arch Intern Med. 1997;157(6):669-675. 
9. CDC. Diabetes Data \& Trends. DHHS Centers for Disease Control and Prevention. [Online] May 10, 2012. [Cited: September 2, 2013.] http://www.cdc.gov/diabetes/statistics/mortalitydka/fNumberOfDKA.htm.

10. Balla U, Malnick S, Schattner A. Early readmissions to the department of medicine as a screening tool for monitoring quality of care problems. Medicine (Baltimore). 2008;87(5):294-300.

11. Rewers A, Chase HP, Mackenzie T, Walravens P, Roback M, Rewers M, Hamman RF, et al. Predictors of acute complications in children with type 1 diabetes. JAMA. 2002;287(19):2511-2518.

12. Barski L, Nevzorov R, Rabaev E, Jotkowitz A, HarmanBoehm I, Zektser M, Zeller L, et al. Diabetic ketoacidosis: clinical characteristics, precipitating factors and outcomes of care. Isr Med Assoc J. 2012;14(5):299-303.

13. Govan L, Wu O, Briggs A, Colhoun HM, Fischbacher
CM, Leese GP, McKnight JA, et al. Achieved levels of HbA1c and likelihood of hospital admission in people with type 1 diabetes in the Scottish population: a study from the Scottish Diabetes Research Network Epidemiology Group. Diabetes Care. 2011;34(9):1992-1997.

14. Isidro ML, Jorge S. Recreational drug abuse in patients hospitalized for diabetic ketosis or diabetic ketoacidosis. Acta Diabetol. 2013;50(2):183-187.

15. Kitabchi AE, Umpierrez GE, Fisher JN, Murphy MB, Stentz FB. Thirty years of personal experience in hyperglycemic crises: diabetic ketoacidosis and hyperglycemic hyperosmolar state. J Clin Endocrinol Metab. 2008;93(5):1541-1552.

16. Nyenwe EA, Loganathan RS, Blum S, Ezuteh DO, Erani DM, Wan JY, Palace MR, et al. Active use of cocaine: an independent risk factor for recurrent diabetic ketoacidosis in a city hospital. Endocr Pract. 2007;13(1):22-29. 